\title{
White noise theory and general improved Kudryashov method for stochastic nonlinear evolution equations with conformable derivatives
}

\section{Abd-Allah Hyder ${ }^{1,2^{*}}$ (B)}

"Correspondence:

abahahmed@kku.edu.sa

'Department of Mathematics,

College of Science, King Khalid

University, Abha, Saudi Arabia

${ }^{2}$ Department of Engineering

Mathematics and Physics, Faculty of

Engineering, Al-Azhar University,

Cairo, Egypt

\section{Springer}

\begin{abstract}
The aim of this work is to investigate the Wick-type stochastic nonlinear evolution equations with conformable derivatives. The general Kudryashov method is improved by a new auxiliary equation. So, a new technique, which we call "the general improved Kudryashov method (GIKM)", is introduced to produce exact solutions for the nonlinear evolution equations with conformable derivatives. By means of GIKM, white noise theory, Hermite transform, and computerized symbolic computation, a novel technique is presented to solve the Wick-type stochastic nonlinear evolution equations with conformable derivatives. This technique is applied to construct exact traveling wave solutions for Wick-type stochastic combined KdV-mKdV equation with conformable derivatives. Moreover, numerical simulations with 3D profiles are shown for the obtained results.
\end{abstract}

MSC: 47J35; 20C40; 83C15

Keywords: Kudryashov method; Combined KdV-mKdV equation; Nonlinear evolution equations; Conformable derivatives; Exact solutions; Stochastic

\section{Introduction}

Nonlinear evolution equations have a significant job in applied sciences, especially in physics. Obtaining traveling wave solutions of these equations has been of major benefit primarily within the context of mathematical physics. Such examinations have prompted many intriguing sorts of solutions in the past, for example, the soliton solutions, the periodic solutions, the cnoidal solutions, the peakon solutions. In any case, searching these solutions has not been simple at all as is showed in the literature. So, many powerful manners have been introduced, such as the homogeneous balance manner [1], the first integral manner [2], the tanh-coth manner [3], the modified tanh-coth manner [4], the inverse scattering manner [5], Hirota's bilinear manner [6], the RB sub-Ode manner [7, 8], the sine-Gordon manner [9], the $\left(G^{\prime} / G\right)$-expansion manner [10], the $\left(G^{\prime} / G, 1 / G\right)$-expansion manner [11], the Exp-function manner [12], F-expansion manner [13, 14], and so on.

(c) The Author(s) 2020. This article is licensed under a Creative Commons Attribution 4.0 International License, which permits use, sharing, adaptation, distribution and reproduction in any medium or format, as long as you give appropriate credit to the original author(s) and the source, provide a link to the Creative Commons licence, and indicate if changes were made. The images or other third party material in this article are included in the article's Creative Commons licence, unless indicated otherwise in a credit line to the material. If material is not included in the article's Creative Commons licence and your intended use is not permitted by statutory regulation or exceeds the permitted use, you will need to obtain permission directly from the copyright holder. To view a copy of this licence, visit http://creativecommons.org/licenses/by/4.0/. 
There are abundant and full treatises related to the fractional and conformable derivatives. Conformable fractional formulation of the fractional calculus was introduced in [15]. The conformable calculus of time-scale was evidenced in [16]. In [17], the fractional traditional mechanics was discussed by some conformable-type derivatives. Lately, the conformable-type differential equations have become a significant object in physics and mathematics. So, abundant experts focus their attention on the analytical and the approximate integrals to these equations $[18,19]$. Existence and uniqueness results for some conformable-type partial differential equations (PDEs) have been proved by Gokdogan et al. [20] and by Sania et al. [21]. In [22], a conformable sub-equation manner was suggested to create exact solutions to the space and time fractional nonlinear resonant Schrödinger equation. Also, fractional modulation to the Nipah virus was given by Markovian process and some local time differential maps [23]. Overall, many studies have been done about the solutions and properties of fractional and conformable-type PDEs [24-29].

Many researchers have been interested in the subject of random traveling wave, it is a very important topic in the field of stochastic partial differential equations (SPDEs). The stochastic KdV equation was proposed by Wadati [30] in 1983. He studied the diffusion of soliton of the equation due to KdV under the Gaussian noise effect. The stochastic traveling wave solutions for the local fractal KdV equation have been obtained by the Expfunction technique in [31] and [32], respectively. Moreover, on account of [14, 33-39], many kinds of Wick-type stochastic and fractional evolution equations have been studied by utilizing diverse expansion techniques and white noise analysis.

Consider a nonlinear PDE (NPDE)

$$
\mathcal{F}\left(\chi, \alpha, u_{\alpha}, u_{\chi}, u_{\chi \chi}, u_{\chi \chi \chi}, \ldots\right)=0 \text {, }
$$

where $(\chi, \alpha) \in \mathbb{R} \times \mathbb{R}_{+}$is the freelance variable and $u(\chi, \alpha)$ is its follower variable. Applying the one-variable transformation

$$
u(\chi, \alpha)=u(\varkappa), \quad \varkappa=\chi-\epsilon \alpha,
$$

we change (1) to an ordinary and nonlinear differential equation (NODE)

$$
\mathcal{G}\left(\varkappa, u, u^{\prime}, u^{\prime \prime}, u^{\prime \prime \prime}, \ldots\right)=0 \text {, }
$$

where $^{\prime}:=\frac{d}{d \varkappa}$. In [40], Kudryashov proposed his manner to find analytical solutions to Eq. (1). He researched for the exact solutions taking into account the expression $u(\varkappa)=$ $\sum_{i=0}^{x} \mu_{i} \mathfrak{X}^{i}$, where $\mathfrak{X}=\frac{1}{1+e^{\varkappa}}$, which is the integral to the equation $\frac{d \mathfrak{X}}{d \varkappa}=\mathfrak{X}^{2}-\mathfrak{X}$. A modified Kudryashov manner was presented by exchanging the ordinary exponential function $e^{\varkappa}$ by means of the general sort of the exponential function $a^{\varkappa}$ in [41-44]. In these contributions, experts got the exact solutions to the NPDE (1) by using the expansion $u(\varkappa)=\sum_{i=0}^{x} \mu_{i} \mathfrak{X}^{i}$, where $\mathfrak{X}=\frac{1}{1 \pm a^{\varkappa}}$, which is the integral to the equation $\frac{d \mathfrak{X}}{d \varkappa}=\ln a\left(\mathfrak{X}^{2}-\mathfrak{X}\right)$. Thereafter, some authors [45-48] applied a general sort of the Kudryashov manner to rummage exact solutions of the NPDE (1). They have selected a rational expansion $u(\varkappa)=$ $\sum_{i=0}^{x} \mu_{i} \mathfrak{X}^{i} / \sum_{j=0}^{y} v_{j} \mathfrak{X}^{j}$, where $\mathfrak{X}=\frac{1}{1+C e^{\varkappa}}$, which is the integral to the equation $\frac{d \mathfrak{X}}{d \varkappa}=\mathfrak{X}^{2}-\mathfrak{X}$. Lately, Abdus Salam and Habiba [49] improved the general Kudryashov manner given in [45] by electing the auxiliary equation $\frac{d \mathfrak{X}}{d \varkappa}=\sigma \mathfrak{X}^{3}-\mathfrak{X}, 0 \neq \sigma \in \mathbb{R}$. This helpful equation has the comprehensive solution $\mathfrak{X}=\frac{ \pm 1}{\sqrt{\sigma+C e^{2 \varkappa}}}$. 
In this work, the general Kudryashov method [45] is improved by the novel auxiliary equation

$$
\mathfrak{X}^{\prime}(\varkappa)=\sigma \mathfrak{X}^{n}(\varkappa)-\mathfrak{X}(\varkappa), \quad 1<n \in \mathbb{N}, 0 \neq \sigma \in \mathbb{R}
$$

which has numerous general solutions depending on the natural number $n$ (see Eq. (25)). Thus, a novel technique to build exact solutions for nonlinear evolution equations is obtained. This technique is called the GIKM. The major feature of the GIKM over the others lies in the way that it utilizes an especially clear and powerful algorithm to obtain exact solutions for large families of nonlinear evolution equations. Also, a large set of exact solutions can be determined effectively on picking the parameters that showed up. Besides, the proposed GIKM generalizes some previous techniques. It depends on improving the general Kudryashov technique by the general auxiliary equation (4), which has various general solutions. Moreover, we apply the GIKM and white noise topics to construct exact solutions for the Wick-type stochastic combined $\mathrm{KdV}-\mathrm{mKdV}$ equation with conformable derivatives. Also, numerical simulations with 3D profiles are provided to the obtained exact solutions.

The remnant of this work is structured as follows: Sect. 2 contains the needed topics about the conformable calculus and the Gaussian analysis of white noise. In Sect. 3, the GIKM is demonstrated. In Sect. 4, we apply the GIKM, jointly with the Gaussian analysis of white noise, to investigate the Wick-type stochastic combined $\mathrm{KdV}-\mathrm{mKdV}$ equation with conformable derivatives. Section 5 gives discussions and numerical simulations for the obtained results. Section 6 presents a conclusion.

\section{Preliminaries}

\subsection{The conformable derivative and integral}

In this division, we recollect the paramount aspects of the conformable-type derivative and its integral.

Definition $2.1([50,51])$ Assume that $\xi$ is a function from $(0, \infty)$ into $\mathbb{R}$. For $\varpi \in(0,1]$, we define the conformable-type derivative of $\xi$ of order $\varpi$ as follows:

$$
D_{\alpha}^{\sigma} \xi(\alpha)=\lim _{h \rightarrow 0} \frac{\xi\left(\alpha+h \alpha^{1-\varpi}\right)-\xi(\alpha)}{h}, \quad \alpha>0
$$

Definition $2.2([50,51])$ Assume that $\xi$ is a $\varpi$-conformable differentiable function for $\alpha \in(0, a), a>0$ and $\lim _{\alpha \rightarrow 0^{+}} D_{\alpha}^{\sigma} \xi(\alpha)$ exists. Then $D_{\alpha}^{\varpi} \xi(0)=\lim _{\alpha \rightarrow 0^{+}} D_{\alpha}^{\varpi} \xi(\alpha)$ and the conformable-type integral of the function $\xi$ beginning from $\alpha_{0} \in[0, \alpha)$ is given by

$$
I^{\varpi, \alpha_{0}} \xi(\alpha)=\int_{\alpha_{0}}^{\alpha} \frac{\xi(\eta)}{\eta^{1-\varpi}} d \eta
$$

where the integral is the classical improper Riemann integral and $\varpi \in(0,1]$.

The coming theorems give some precious properties for the conformable-type derivative. 
Theorem 2.1 ([50,51]) Assume that $\varpi \in(0,1], \xi$ and $\zeta$ are $\varpi$-order conformable differentiable functions at $\alpha \in(0, \infty)$, and $\xi$ is differentiable (in the usual sense) with respect to $\alpha$. Then:

(i) $D_{\alpha}^{\sigma}(a \xi+b \zeta)=a D_{\alpha}^{\varpi} \xi+b D_{\alpha}^{\sigma} \zeta$ for all $a, b \in \mathbb{R}$;

(ii) $D_{\alpha}^{\varpi}\left(\alpha^{a}\right)=a \alpha^{a-\infty}$ for all $a \in \mathbb{R}$;

(iii) $D_{\alpha}^{\varpi}(\xi \zeta)=\xi D_{\alpha}^{\varpi} \zeta+\zeta D_{\alpha}^{\sigma} \xi$;

(iv) $D_{\alpha}^{\sigma}\left(\frac{\xi}{\zeta}\right)=\frac{\zeta D_{\alpha}^{\Phi} \xi-\xi D_{\alpha}^{\Phi} \zeta}{\zeta^{2}}$;

(v) $D_{\alpha}^{\varpi}(\xi(\alpha))=\alpha^{1-\varpi} \xi^{\prime}(\alpha)$,

where' denotes the usual derivative with regard to $\alpha$.

Theorem 2.2 ([44]) Assume that the function $\xi$ is a differentiable and $\varpi$-order conformable differentiable function on $(0, \infty)$. Also, assume that $\zeta$ is a differentiable function defined on the range of $\xi$. Then

$$
D_{\alpha}^{\varpi}(\xi \circ \zeta)(\alpha)=\alpha^{1-\varpi}[\zeta(\alpha)]^{\varpi-1} \zeta^{\prime}(\alpha)\left(D_{\alpha}^{\varpi} \xi(\alpha)\right)_{\alpha=\zeta} .
$$

\subsection{Basilar topics of white noise discipline}

The Gaussian white noise discipline begins with the rigging $\mathcal{D}\left(\mathbb{R}^{N}\right) \subset L^{2}\left(\mathbb{R}^{N}\right) \subset \mathcal{D}^{*}\left(\mathbb{R}^{N}\right)$ where $\mathcal{D}\left(\mathbb{R}^{N}\right)$ is the Schwartz space of quickly decreasing, unlimited differentiable functions on $\mathbb{R}^{N}$ and $\mathcal{D}^{*}\left(\mathbb{R}^{N}\right)$ is the tempered space of distributions. Depending on the Bochner-Minlos theorem [52], we have a lonesome white noise measure $\mu_{w}$ on $\left(\mathcal{D}^{*}\left(\mathbb{R}^{N}\right), \beta\left(\mathcal{D}^{*}\left(\mathbb{R}^{N}\right)\right)\right)$. Presume that $\zeta_{n}(x)=\pi^{-1 / 4}((n-1) !)^{-1 / 2} e^{-x^{2} / 2} h_{n-1}(\sqrt{2 x}), n \in \geq 1$ are the Hermite functions, where $h_{n}(x)$ denotes the Hermite polynomials. It is well known that the gathering $\left(\zeta_{n}\right)_{n \in \mathbb{N}}$ fabricates an orthonormal basis for $L^{2}(\mathbb{R})$. Let $m=\left(m_{1}, \ldots, m_{N}\right)$ be $N$-dimensional multi-indices with $m_{1}, \ldots, m_{N} \in \mathbb{N}$, then the tensor multiplications $\zeta_{m}:=\zeta_{\left(m_{1}, \ldots, m_{N}\right)}=\chi_{m_{1}} \otimes \cdots \otimes \chi_{m_{N}}, m \in \mathbb{N}^{N}$ fabricate an orthonormal basis to $L^{2}\left(\mathbb{R}^{N}\right)$. Introduce an ordering in $\mathbb{N}^{N}$ by

$$
i<j \Rightarrow \sum_{k=1}^{N} m_{k}^{(i)} \leq \sum_{k=1}^{N} m_{k}^{(j)}, \quad \text { where } m^{(i)}=\left(m_{k}^{(i)}\right)_{k=1}^{N}, m^{(j)}=\left(m_{k}^{(j)}\right)_{k=1}^{N} \in \mathbb{N}^{N}
$$

Using the above ordering, we define $\varrho_{i}:=\zeta_{m^{(i)}}=\zeta_{m_{1}^{(i)}} \otimes \cdots \otimes \zeta_{m_{N}^{(i)}}, i \in \mathbb{N}$. Let $\mathbb{J}=\left(\mathbb{N}_{0}^{\mathbb{N}}\right)_{c}$ be the aggregate of sequences $m=\left(m_{i}\right)_{i \in \mathbb{N}}$ with compact support and $m_{i} \geq 1$. For $m \in \mathbb{J}$, we set

$$
\mathbb{H}_{m}(\vartheta)=\prod_{i=1}^{\infty} h_{m_{i}}\left(\left\langle\vartheta, \varrho_{i}\right\rangle\right), \quad \vartheta \in \mathcal{D}^{*}\left(\mathbb{R}^{N}\right)
$$

Let $n \in \mathbb{N}$, the Kondrative space of test stochastic functions $(\mathcal{D})_{1}^{n}$ is defined by

$$
\begin{aligned}
(\mathcal{D})_{1}^{n}= & \left\{f=\sum_{m} c_{m} \mathbb{H}_{m} \in \bigoplus_{k=1}^{n} L^{2}\left(\mu_{w}\right): c_{m} \in \mathbb{R}^{n}\right. \text { and } \\
& \left.\|f\|_{1, k}^{2}:=\sum_{m} c_{m}^{2}(m !)^{2}(2 \mathbb{N})^{k m}<\infty \forall k \in \mathbb{N}\right\},
\end{aligned}
$$


and the Kondrative space of distribution stochastic functions $(\mathcal{D})_{-1}^{n}$ is defined by

$$
\begin{aligned}
(\mathcal{D})_{-1}^{n}= & \left\{F=\sum_{m} b_{m} \mathbb{H}_{m}: b_{m} \in \mathbb{R}^{n}\right. \text { and } \\
& \left.\|F\|_{-1, k}^{2}:=\sum_{m} b_{m}^{2}(2 \mathbb{N})^{-q m}<\infty \text { for some } q \in \mathbb{N}\right\} .
\end{aligned}
$$

The Wick product of two stochastic distributions $F=\sum_{m} a_{m} \mathbb{H}_{m}, G=\sum_{\bar{m}} b_{\bar{m}} \mathbb{H}_{\bar{m}} \in(\mathcal{D})_{-1}^{n}$ with $a_{m}, b_{\bar{m}} \in \mathbb{R}^{n}$ is known as

$$
F \diamond G=\sum_{m, \bar{m}}\left(a_{m}, b_{\bar{m}}\right) \mathbb{H}_{m+\bar{m}} .
$$

Let $F=\sum_{m} a_{m} \mathbb{H}_{m} \in(\mathcal{D})_{-1}^{n}$ with $a_{m} \in \mathbb{R}^{n}$. The Hermite transform of $F$ is defined by

$$
\mathcal{H} F(z)=\widetilde{F}(z)=\sum_{m} a_{m} z^{m} \in \mathbb{C}^{n} \quad \text { (when convergent), }
$$

where $z=\left(z_{1}, z_{2}, \ldots\right) \in \mathbb{C}^{\mathbb{N}}$ and $z^{m}=\prod_{i=1}^{\infty} z_{i}^{m_{i}}$, with $m=\left(m_{1}, m_{2}, \ldots\right) \in \mathbb{J}$ and $z_{i}^{0}=1$.

For $F, G \in(\mathcal{D})_{-1}^{n}$, via the shape of Hermite transformation, we have

$$
\widetilde{F \diamond G}(z)=\widetilde{F}(z) \bullet \widetilde{G}(z)
$$

for all $z$ such that $\widetilde{F}(z)$ and $\widetilde{G}(z)$ exist. The relation "•" indicates the bilinear multiplication in $\mathbb{C}^{n}$, which is known as $\left(z_{1}^{1}, \ldots, z_{n}^{1}\right) \bullet\left(z_{1}^{2}, \ldots, z_{n}^{2}\right)=\sum_{i=1}^{n} z_{i}^{1} z_{i}^{2}$, where $z_{i}^{k} \in \mathbb{C}$. Thus, the Hermite transform changes the Wick multiplication into the classical multiplication and convergence in $(\mathcal{D})_{-1}^{n}$ into bounded and pointwise convergence in a certain neighborhood of the origin in $\mathbb{C}^{n}$. For more specifics about Kondrative spaces, Hermite transformation, and Wick multiplication, we refer to [52].

In the following, the distribution stochastic process (or $(\mathcal{D})_{-1}^{n}$-process) is a measurable map $u$ from $\mathbb{R}^{N}$ into $(\mathcal{D})_{-1}^{n}$. Furthermore, if the $(\mathcal{D})_{-1}^{n}$-valued function $u$ is continuous, differentiable, $C^{k}$, etc., then the $(\mathcal{D})_{-1}^{n}$-process $u$ has the same features, respectively. Now, for $\pi<\infty, \rho>0$, deem the infinite dimensional neighborhoods $\mathcal{O}_{\pi}(\rho)=\left\{\left(z_{1}, z_{2}, \ldots\right) \in \mathbb{C}^{\mathbb{N}}\right.$ : $\left.\sum_{m \neq 0}\left|z^{m}\right|^{2}(2 \mathbb{N})^{\pi m}<\rho^{2}\right\}$ of the origin in $\mathbb{C}^{\mathbb{N}}$ [52]. To study the stochastic conformable PDEs, we require the following facts.

Lemma $2.1([52,53])$ Suppose that $X(\alpha, \vartheta)$ and $Y(\alpha, \vartheta)$ are $(\mathcal{D})_{-1}$-processes such that

(i) $D_{\alpha}^{\sigma} \tilde{X}(\alpha, z)=\tilde{Y}(\alpha, z) \forall(\alpha, z) \in(s, t) \times \mathcal{O}_{\pi}(\rho)$ and that

(ii) $\tilde{Y}(\alpha, z)$ is a bounded function for $(\alpha, z) \in(s, t) \times \mathcal{O}_{\pi}(\rho)$ and continuous for $\alpha \in(s, t)$ $\forall z \in \mathcal{O}_{\pi}(\rho)$.

Then $X(\alpha, \vartheta)$ has a $\varpi$-order conformable derivative for each $\alpha \in(s, t)$ and

$$
D_{\alpha}^{\varpi} X(\alpha, \vartheta)=Y(\alpha, \vartheta) \quad \text { in }(\mathcal{D})_{-1}
$$

Lemma $2.2([52,53])$ Let $X(\alpha, \vartheta)$ be a $(\mathcal{D})_{-1}$-process. Suppose that there exist $\pi<\infty, \rho>0$ such that

$$
\sup \left\{\tilde{X}(\alpha, z): \alpha \in[s, t], z \in \mathcal{O}_{\pi}(\rho)\right\}<\infty
$$


and $\widetilde{X}(\alpha, z)$ is a continuous function for $\alpha \in[s, t] \forall z \in \mathcal{O}_{\pi}(\rho)$. Then the $\varpi$-order conformable integral operator of $X(\alpha)$ exists and

$$
\widetilde{I^{\varpi, s} X(\alpha)}(z)=I^{\varpi, s} \widetilde{X}(\alpha, z) \quad \text { for } \varpi, s \geq 0, \alpha \in[s, t], z \in \mathcal{O}_{\pi}(\rho) .
$$

Theorem 2.3 ([52]) Suppose that $u(\chi, \alpha, z)$ is a solution (in the usual strong and pointwise sense) of the equation

$$
\widetilde{\Omega}\left(u, \chi, \alpha, z, D_{\alpha}^{\sigma}, D_{\chi_{1}}^{\sigma}, \ldots, D_{\chi_{N}}^{\sigma}\right)=0
$$

for $(\chi, \alpha)$ in some bounded open set $\mathbf{D} \subset \mathbb{R}^{N} \times \mathbb{R}_{+}, \forall z \in \mathcal{O}_{\pi}(\rho)$ and for $\pi<\infty \rho>0$. Moreover, suppose that $u(\chi, \alpha, z)$ and all its conformable derivatives, which are implicated in Eq. (18), are bounded for $(\chi, \alpha, z) \in \mathbf{D} \times \mathcal{O}_{\pi}(\rho)$, continuous for $(\chi, \alpha) \in \mathbf{D} \forall z \in \mathcal{O}_{\pi}(\rho)$, and analytic $\forall z \in \mathcal{O}_{\pi}(\rho)$ for all $(\chi, \alpha) \in \mathbf{D}$. Then $\exists U(\chi, \alpha) \in(\mathcal{D})_{-1}$ such that $u(\chi, \alpha, z)=$ $\tilde{U}(\chi, \alpha)(z)$ for all $(\chi, \alpha, z) \in \mathbf{D} \times \mathcal{O}_{\pi}(\rho)$ and $U(\chi, \alpha)$ solves (in the strong sense) the equation

$$
\Omega^{\diamond}\left(U, \chi, \alpha, \vartheta, D_{\alpha}^{\sigma}, D_{\chi_{1}}^{\sigma}, \ldots, D_{\chi_{N}}^{\sigma}\right)=0 \quad \text { in }(\mathcal{D})_{-1} .
$$

\section{Demonstration of the GIKM}

Consider a conformable NPDE in the form

$$
\mathcal{P}\left(u, \chi, \alpha, D_{\alpha}^{\varpi} u, D_{\chi}^{\varpi} u, D_{\chi}^{2 \pi} u, D_{\chi}^{3 \pi} u, \ldots\right)=0
$$

where $u=u(\chi, \alpha)$ is the unknown function and $\mathcal{P}$ is a polynomial function in $u$ and its conformable derivatives. To obtain wave solution for Eq. (20), we use the wave transformation

$$
u=u(\varkappa), \quad \varkappa(\chi, \alpha)=\frac{\chi^{\varpi}}{\varpi}+\omega \int_{a}^{\alpha} \frac{\theta(\eta)}{\eta^{1-\varpi}} d \eta
$$

where $a \geq 0, \omega$ are constants and $\theta$ is a nonzero function to be determined later. Hence, Eq. (21) converts Eq. (20) to a NODE

$$
\mathcal{Q}\left(u, \varkappa, \frac{d u}{d \varkappa}, \frac{d^{2} u}{d \varkappa^{2}}, \frac{d^{3} u}{d \varkappa^{3}}, \ldots\right)=0
$$

For easiness, we integrate Eq. (22) as long as all terms involve derivatives. Then, we equalize the integration constants to zero. Thereafter, the solution of Eq. (22) can be expanding as the form

$$
u(\varkappa)=\frac{\sum_{i=0}^{x} \mu_{i}(\alpha) \mathfrak{X}^{i}(\varkappa)}{\sum_{j=0}^{y} v_{j}(\alpha) \mathfrak{X}^{j}(\varkappa)},
$$

where $\mu_{i}, v_{j}(i=0,1, \ldots, x, j=0,1, \ldots, y)$ are functions to be determined and $\mathfrak{X}$ solves the common auxiliary equation

$$
\mathfrak{X}^{\prime}(\varkappa)=\sigma \mathfrak{X}^{n}(\varkappa)-\mathfrak{X}(\varkappa), \quad 1<n \in \mathbb{N}, 0 \neq \sigma \in \mathbb{R} .
$$


Solving Eq. (24) gives a general family of solutions

$$
\mathfrak{X}(\varkappa)= \begin{cases}\frac{1}{\sqrt[n-1]{\sigma+C \exp [(n-1) \varkappa]},} & n=2,4,6,8, \ldots, \\ \frac{ \pm 1}{\sqrt[n-1]{\sigma+C \exp [(n-1) \varkappa]}}, & n=3,7,11,15, \ldots \\ \frac{ \pm 1}{\sqrt[n-1]{\sigma+C \exp [(n-1) \varkappa]}}, & n=5,9,13,17, \ldots\end{cases}
$$

The integer numbers $x$ and $y$ can be appointed by balancing the highest order linear and nonlinear terms in Eq. (22). By inserting Eqs. (23) and (24) into Eq. (22), we get an algebraic-form equation in $\mathfrak{X}$ and its powers. Placing the coefficients of all terms that include the similar power for $\mathfrak{X}$ to be zero, gives a system of algebraic-form equations in $\mu_{i}$, $v_{j}$, and $\theta$. By employing the symbolic system Mathematica, we can determine $\mu_{i}, v_{j}$, and $\theta$. Lastly, by utilizing these values and Eq. (25), we can construct some exact and traveling wave solutions to Eq. (20).

\section{Application to Wick-type stochastic combined KdV-mKdV equation with conformable derivatives}

In this section, GIKM for $n=5$, white noise theory, Hermite transform, and computerized symbolic computation are applied to find exact traveling wave solutions of Wicktype stochastic combined $\mathrm{KdV}-\mathrm{mKdV}$ with conformable derivatives. The $\mathrm{KdV}$ and $\mathrm{mKdV}$ equations are solitary equations, which have been widely researched. For these equations, the nonlinear terms usually arise in abundant physical issues, like flow mechanics, quantum fields, and plasma physics. This section is devoted to constructing exact traveling wave solutions of Wick-type stochastic combined $\mathrm{KdV}-\mathrm{mKdV}$ equation with conformable derivatives

$$
D_{\alpha}^{\varpi} U+\Delta(\alpha) \diamond U \diamond D_{\chi}^{\varpi} U+\Lambda(\alpha) \diamond U^{\diamond 2} \diamond D_{\chi}^{\varpi} U+D_{\chi}^{3 \varpi} U=0
$$

where $(\chi, \alpha) \in \mathbb{R} \times \mathbb{R}_{+}$and $0<\varpi \leq 1$, while $\Delta$ and $\Lambda$ are real and integrable nonzero functions with values in $(\mathcal{D})_{-1}$. Equation (26) is the perturbation of the variable coefficients combined $\mathrm{KdV}-\mathrm{mKdV}$ equation with conformable derivatives

$$
D_{\alpha}^{\varpi} u+\delta(\alpha) u D_{\chi}^{\varpi} u+\lambda(\alpha) u^{2} D_{\chi}^{\varpi} u+D_{\chi}^{3 \varpi} u=0
$$

where $\delta, \lambda$ are nonzero integrable functions on $\mathbb{R}_{+}$. Moreover, if Eq. (27) is considered in some random ambience, we have a random combined $\mathrm{KdV}-\mathrm{mKdV}$ equation. To construct exact solutions of the random combined $\mathrm{KdV}-\mathrm{mKdV}$ equation, we only examine it in a white noise ambience, thus, we will investigate the Wick-type stochastic combined KdVmKdV equation (26).

By using Hermite transform and Eq. (26), we get a conformable deterministic equation

$$
\begin{aligned}
& D_{\alpha}^{\varpi} \widetilde{U}(\chi, \alpha, z)+\widetilde{\Delta}(\alpha, z) \widetilde{U}(\chi, \alpha, z) D_{\chi}^{\varpi} \widetilde{U}(\chi, \alpha, z) \\
& \quad+\widetilde{\Lambda}(\alpha, z) \widetilde{U}^{2} D_{\chi}^{\varpi} \widetilde{U}(\chi, \alpha, z)+D_{\chi}^{3 \sigma} \widetilde{U}(\chi, \alpha, z)=0,
\end{aligned}
$$

where $z=\left(z_{1}, z_{2}, \ldots\right) \in\left(\mathbb{C}^{\mathbb{N}}\right)_{c}$. To construct traveling wave solutions to Eq. (28), we employ the transformations $\widetilde{\Delta}(\alpha, z)=\delta(\alpha, z), \widetilde{\Lambda}(\alpha, z)=\lambda(\alpha, z), \widetilde{U}(\chi, \alpha, z)=u(\chi, \alpha, z)=u(\varkappa(\chi, \alpha, z))$ 
with

$$
\varkappa(\chi, \alpha, z)=\frac{\chi^{\varpi}}{\varpi}+\omega \int_{a}^{\alpha} \frac{\theta(\eta, z)}{\eta^{1-\varpi}} d \eta
$$

where $\omega$ is a free constant and $\theta$ is a nonzero function to be specified. Hence, Eq. (28) can be transformed to the following NODE

$$
\omega \theta \frac{d u}{d \varkappa}+\delta u \frac{d u}{d \varkappa}+\lambda u^{2} \frac{d u}{d \varkappa}+\frac{d^{3} u}{d \varkappa^{3}}=0
$$

Integrating the NODE (30) and placing the integration constants to be zero give

$$
\omega \theta u+\frac{\delta}{2} u^{2}+\frac{\lambda}{3} u^{3}+\frac{d^{2} u}{d \varkappa^{2}}=0
$$

Considering the homogeneous balance for $\frac{d^{2} u}{d \varkappa^{2}}$ and $u^{3}$, we get $x-y-4=0$. Let $y=1$, then $x=5$. So, we can set the wave solution of Eq. (31) as the form

$$
u(\varkappa(\chi, \alpha, z))=\frac{\sum_{i=0}^{5} \mu_{i}(\alpha, z) \mathfrak{X}^{i}(\varkappa(\chi, \alpha, z))}{\sum_{j=0}^{1} v_{j}(\alpha, z) \mathfrak{X}^{j}(\varkappa(\chi, \alpha, z))} .
$$

Substituting Eqs. (32) and (24) for $n=5$ into Eq. (31) gives an algebraic-form equation in $\mathfrak{X}$ and its powers. Equating the coefficients of the terms that contain the same power for $\mathfrak{X}$ to zero gives a system of algebraic-form equations in $\mu_{i}, v_{j}(i=0, \ldots, 5, j=0,1)$ and $\theta$ (see the Appendix). By treating this system via Mathematica, we obtain the following sets of values.

Case I.

$$
\left\{\begin{array}{l}
\mu_{0}=\mu_{0}, \quad \mu_{1}=\frac{36 \mu_{0} v_{0}^{2} \nu_{1}-180 \mu_{0}^{2} \nu_{0} \nu_{1} \delta+12 \mu_{0}^{2} \nu_{1}\left(3 v_{0} \delta+2 \mu_{0} \lambda\right)}{6\left(\mu_{0}^{2} \lambda+v_{0}^{2}+\mu_{0} v_{0} \delta\right)-\mu_{0}\left(3 v_{0} \delta+2 \mu_{0} \lambda\right)}, \\
\mu_{2}= \pm \frac{155 v_{0}^{3}}{102 v_{1}^{2} \sqrt{6 \lambda}}, \quad \mu_{3}= \pm \frac{10 v_{0}^{2}}{v_{1} \sqrt{96 \lambda}}, \quad \mu_{4}=0, \quad \mu_{5}= \pm \frac{\sqrt{96} v_{1} \sigma}{\sqrt{\lambda}} \\
v_{0}=v_{0}, \quad \nu_{1}=v_{1}, \quad \theta=\frac{\mu_{0}\left(3 v_{0} \delta+2 \mu_{0} \lambda\right)}{6 \omega \nu_{0}^{2}},
\end{array}\right.
$$

where $\mu_{0}, v_{0}$, and $v_{1}$ are free integrable functions on $\mathbb{R}_{+}$. Substituting the values (33) into (32) and using (25) produce traveling wave solutions to Eq. (28) as follows:

$$
\begin{aligned}
u_{1}(\chi, \alpha, z)= & \frac{\sqrt[4]{\sigma+C \exp [4 \varkappa(\chi, \alpha, z)]}}{v_{1}(\alpha, z) \pm v_{0}(\alpha, z) \sqrt[4]{\sigma+C \exp [4 \varkappa(\chi, \alpha, z)]}}\left[\frac{\mu_{0}(\alpha, z)+A(\alpha, z)}{102 v_{1}^{2}(\alpha, z) \sqrt{6 \lambda(\alpha, z)}}\right. \\
& \pm \frac{155 v_{0}^{3}(\alpha, z)}{(\sigma+C \exp [4 \varkappa(\chi, \alpha, z)])^{\frac{1}{2}}}+\frac{255 v_{1}^{3}(\alpha, z)}{(\sigma+C \exp [4 \varkappa(\chi, \alpha, z)])^{\frac{3}{4}}} \\
& \left.+\frac{2448 \sigma v_{1}^{3}(\alpha, z)}{(\sigma+C \exp [4 \varkappa(\chi, \alpha, z)])^{\frac{5}{4}}}\right] \\
u_{2}(\chi, \alpha, z)= & \frac{\sqrt[4]{\sigma+C \exp [4 \varkappa(\chi, \alpha, z)]}}{v_{1}(\alpha, z) \pm i v_{0}(\alpha, z) \sqrt[4]{\sigma+C \exp [4 \varkappa(\chi, \alpha, z)]}}\left[\frac{\mu_{0}(\alpha, z)+A(\alpha, z)}{102 v_{1}^{2}(\alpha, z) \sqrt{6 \lambda(\alpha, z)}}\right.
\end{aligned}
$$




$$
\begin{aligned}
& \pm i \frac{155 v_{0}^{3}(\alpha, z)}{(\sigma+C \exp [4 \varkappa(\chi, \alpha, z)])^{\frac{1}{2}}} \pm i \frac{255 v_{1}^{3}(\alpha, z)}{(\sigma+C \exp [4 \varkappa(\chi, \alpha, z)])^{\frac{3}{4}}} \\
& \left. \pm i \frac{2448 \sigma v_{1}^{3}(\alpha, z)}{(\sigma+C \exp [4 \varkappa(\chi, \alpha, z)])^{\frac{5}{4}}}\right]
\end{aligned}
$$

where

$$
A(\alpha, z)=\frac{\left(\begin{array}{c}
36 \mu_{0}(\alpha, z) v_{0}^{2}(\alpha, z) v_{1}(\alpha, z)-180 \mu_{0}^{2}(\alpha, z) v_{0}(\alpha, z) v_{1}(\alpha, z) \delta(\alpha, z) \\
+12 \mu_{0}^{2}(\alpha, z) v_{1}(\alpha, z)\left(3 v_{0}(\alpha, z) \delta(\alpha, z)+2 \mu_{0}(\alpha, z) \lambda(\alpha, z)\right)
\end{array}\right)}{\left(\begin{array}{c}
6\left(\mu_{0}^{2}(\alpha, z) \lambda(\alpha, z)+v_{0}^{2}(\alpha, z)+\mu_{0}(\alpha, z) v_{0}(\alpha, z) \delta(\alpha, z)\right) \\
-\mu_{0}(\alpha, z)\left(3 v_{0}(\alpha, z) \delta(\alpha, z)+2 \mu_{0}(\alpha, z) \lambda(\alpha, z)\right)
\end{array}\right)}
$$

and

$$
\varkappa(\chi, \alpha, z)=\frac{\chi^{\varpi}}{\varpi}+\int_{a}^{\alpha} \frac{\mu_{0}(\eta, z)\left(3 v_{0}(\eta, z) \delta(\eta, z)+2 \mu_{0}(\eta, z) \lambda(\eta, z)\right)}{6 \eta^{1-\varpi} v_{0}^{2}(\eta, z)} d \eta
$$

provided that $\lambda>0$ and $\nu_{0} \neq 0$.

Case II.

$$
\left\{\begin{array}{lll}
\mu_{0}=\frac{v_{0} \sqrt{9 \delta^{2}-384 \lambda}-3 v_{0} \delta}{8 \lambda}, & \mu_{1}=\frac{\nu_{1} \sqrt{3 \delta^{2}-128 \lambda}-3 v_{1} \delta}{8 \lambda}, \\
\mu_{2}=\frac{\mu_{4}\left(813 v_{0}^{2} \sigma^{2}-8 \mu_{4}^{2} \lambda\right)}{120 v_{1}^{2} \sigma^{2}}, & \mu_{3}=\frac{-306 \mu_{4} v_{0}}{72 v_{1}}, \quad \mu_{4}=\mu_{4}, \quad \mu_{5}=0 \\
\nu_{0}=v_{0}, \quad \nu_{1}=v_{1}, & \theta=\frac{\delta\left(3 \delta-\sqrt{9 \delta^{2}-384 \lambda}\right)-64 \lambda}{32 \omega \lambda},
\end{array}\right.
$$

where $\mu_{4}, v_{0}$, and $v_{1}$ are free integrable functions on $\mathbb{R}_{+}$. Substituting the values (38) into (32) and using (25) produce traveling wave solutions to Eq. (28) as follows:

$$
\begin{aligned}
u_{3}(\chi, \alpha, z)= & \frac{\sqrt[4]{\sigma+C \exp [4 \varkappa(\chi, \alpha, z)]}}{v_{1}(\alpha, z) \pm v_{0}(\alpha, z) \sqrt[4]{\sigma+C \exp [4 \varkappa(\chi, \alpha, z)]}} \\
& \times\left[\frac{\left(v_{0}(\alpha, z) \sqrt{9 \delta^{2}(\alpha, z)-384 \lambda(\alpha, z)}-3 v_{0}(\alpha, z) \delta(\alpha, z)\right)}{8 \lambda(\alpha, z)}\right. \\
& \pm \frac{v_{1}(\alpha, z) \sqrt{3 \delta^{2}(\alpha, z)-128 \lambda(\alpha, z)}-3 v_{1}(\alpha, z) \delta(\alpha, z)}{8 \lambda(\alpha, z)(\sigma+C \exp [4 \varkappa(\chi, \alpha, z)])^{\frac{1}{4}}} \\
& +\frac{\mu_{4}(\alpha, z)\left(813 v_{0}^{2}(\alpha, z) \sigma^{2}-8 \mu_{4}^{2}(\alpha, z) \lambda(\alpha, z)\right)}{120 v_{1}^{2}(\alpha, z) \sigma^{2}(\sigma+C \exp [4 \varkappa(\chi, \alpha, z)])^{\frac{1}{2}}} \\
& \pm \frac{306 \mu_{4}(\alpha, z) v_{0}(\alpha, z)}{72 v_{1}(\alpha, z)(\sigma+C \exp [4 \varkappa(\chi, \alpha, z)])^{\frac{3}{4}}} \\
& \left.+\frac{\mu_{4}(\alpha, z)}{(\sigma+C \exp [4 \varkappa(\chi, \alpha, z)])}\right], \\
u_{4}(\chi, \alpha, z)= & \frac{\sqrt[4]{\sigma+C \exp [4 \varkappa(\chi, \alpha, z)]}}{v_{1}(\alpha, z) \pm i v_{0}(\alpha, z) \sqrt[4]{\sigma+C \exp [4 \varkappa(\chi, \alpha, z)]}} \\
& \times\left[\frac{\left(v_{0}(\alpha, z) \sqrt{9 \delta^{2}(\alpha, z)-384 \lambda(\alpha, z)}-3 v_{0}(\alpha, z) \delta(\alpha, z)\right)}{8 \lambda(\alpha, z)}\right.
\end{aligned}
$$




$$
\begin{aligned}
& \pm i \frac{\nu_{1}(\alpha, z) \sqrt{3 \delta^{2}(\alpha, z)-128 \lambda(\alpha, z)}-3 v_{1}(\alpha, z) \delta(\alpha, z)}{8 \lambda(\alpha, z)(\sigma+C \exp [4 \varkappa(\chi, \alpha, z)])^{\frac{1}{4}}} \\
& +\frac{\mu_{4}(\alpha, z)\left(813 v_{0}^{2}(\alpha, z) \sigma^{2}-8 \mu_{4}^{2}(\alpha, z) \lambda(\alpha, z)\right)}{120 v_{1}^{2}(\alpha, z) \sigma^{2}(\sigma+C \exp [4 \varkappa(\chi, \alpha, z)])^{\frac{1}{2}}} \\
& \pm i \frac{306 \mu_{4}(\alpha, z) v_{0}(\alpha, z)}{72 v_{1}(\alpha, z)(\sigma+C \exp [4 \varkappa(\chi, \alpha, z)])^{\frac{3}{4}}} \\
& \left.+\frac{\mu_{4}(\alpha, z)}{(\sigma+C \exp [4 \varkappa(\chi, \alpha, z)])}\right]
\end{aligned}
$$

where

$$
\varkappa(\chi, \alpha, z)=\frac{\chi^{\varpi}}{\varpi}+\int_{a}^{\alpha} \frac{\delta(\eta, z)\left(3 \delta(\eta, z)-\sqrt{9 \delta^{2}(\eta, z)-384 \lambda(\eta, z)}\right)-64 \lambda(\eta, z)}{32 \eta^{1-\varpi} \lambda(\eta, z)} d \eta,
$$

provided that $\lambda \neq 0, \sigma \neq 0, v_{1} \neq 0$, and $\delta^{2} \geq \frac{128 \lambda}{3}$.

Case III.

$$
\left\{\begin{array}{l}
\mu_{0}=\mu_{0}, \quad \mu_{1}=\frac{v_{1}\left(-3 \delta+\sqrt{9 \delta^{2}-528 \lambda}\right)}{8 \lambda}, \quad \mu_{2}=\mu_{3}=\mu_{4}=\mu_{5}=0 \\
v_{0}=-\frac{\mu_{0}}{66}\left(3 \delta+\sqrt{9 \delta^{2}-528 \lambda}\right), \quad v_{1}=v_{1}, \\
\theta=\frac{-3 \delta^{2}+\delta \sqrt{9 \delta^{2}-528 \lambda}-88 \lambda}{32 \omega \lambda},
\end{array}\right.
$$

where $\mu_{0}$ and $\nu_{1}$ are free integrable functions on $\mathbb{R}_{+}$. Substituting the values (42) into (32) and using (25) produces traveling wave solutions to Eq. (28) as follows:

$$
\begin{gathered}
u_{5}(\chi, \alpha, z)=\frac{\left(\begin{array}{c}
528 \mu_{0}(\alpha, z) \lambda(\alpha, z)(\sigma+C \exp [4 \varkappa(\chi, \alpha, z)])^{\frac{1}{4}} \\
\pm 66 v_{1}(\alpha, z)\left(-3 \delta(\alpha, z)+\sqrt{9 \delta^{2}(\alpha, z)-528 \lambda(\alpha, z)}\right)
\end{array}\right)}{\left(\begin{array}{c}
-8 \mu_{0}(\alpha, z) \lambda(\alpha, z)\left(3 \delta(\alpha, z)+\sqrt{9 \delta^{2}(\alpha, z)-528 \lambda(\alpha, z)}\right) \\
\times(\sigma+C \exp [4 \varkappa(\chi, \alpha, z)])^{\frac{1}{4}} \pm 528 v_{1}(\alpha, z) \lambda(\alpha, z)
\end{array}\right)} \\
u_{6}(\chi, \alpha, z)=\frac{\left(\begin{array}{c}
528 \mu_{0}(\alpha, z) \lambda(\alpha, z)(\sigma+C \exp [4 \varkappa(\chi, \alpha, z)])^{\frac{1}{4}} \\
\pm 66 i v_{1}(\alpha, z)\left(-3 \delta(\alpha, z)+\sqrt{9 \delta^{2}(\alpha, z)-528 \lambda(\alpha, z)}\right)
\end{array}\right)}{\left(\begin{array}{r}
-8 \mu_{0}(\alpha, z) \lambda(\alpha, z)\left(3 \delta(\alpha, z)+\sqrt{9 \delta^{2}(\alpha, z)-528 \lambda(\alpha, z)}\right) \\
\times(\sigma+C \exp [4 \varkappa(\chi, \alpha, z)])^{\frac{1}{4}} \pm 528 i v_{1}(\alpha, z) \lambda(\alpha, z)
\end{array}\right)},
\end{gathered}
$$

where

$$
\varkappa(\chi, \alpha, z)=\frac{\chi^{\varpi}}{\varpi}+\int_{a}^{\alpha} \frac{-3 \delta^{2}(\eta, z)+\delta(\eta, z) \sqrt{9 \delta^{2}(\eta, z)-528 \lambda(\eta, z)}-88 \lambda(\eta, z)}{32 \eta^{1-\varpi} \lambda(\eta, z)} d \eta
$$

provided that $\lambda \neq 0$ and $\delta^{2} \geq \frac{528 \lambda}{9}$.

Obviously, we can find different traveling wave solutions of Eq. (28) by applying different cases to the solutions of the algebraic system in the Appendix.

The features of exponential functions lead to the existence of an open bounded set $\mathbf{D} \subset$ $\mathbb{R} \times \mathbb{R}_{+}, \pi<\infty, \rho>0$ provided that the solution $u(\chi, \alpha, z)$ of Eq. (28) and all its conformable derivatives that are included in Eq. (28) are uniformly bounded with respect to $(\chi, \alpha, z) \in$ $\mathbf{D} \times \mathcal{O}_{\pi}(\rho)$, continuous for $(\chi, \alpha) \in \mathbf{D} \forall z \in \mathcal{O}_{\pi}(\rho)$, and analytic for $z \in \mathcal{O}_{\pi}(\rho) \forall(\chi, \alpha) \in \mathbf{D}$. 
According to Theorem 2.3, $\exists U(\chi, \alpha) \in(\mathcal{D})_{-1}$ such that $u(\chi, \alpha, z)=\widetilde{U}(\chi, \alpha)(z) \forall(\chi, \alpha, z) \in$ $\mathbf{D} \times \mathcal{O}_{\pi}(\rho)$ and $U(\chi, \alpha)$ solves Eq. $(26)$ in $(\mathcal{D})_{-1}$. Hence, by taking the inverse Hermite transform to Eqs. (34)-(37), (39)-(41), and (43)-(45), we obtain the stochastic solutions of Eq. (26) as follows:

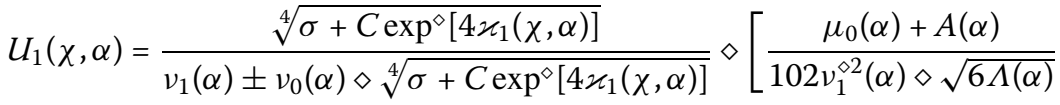

$$
\begin{aligned}
& \pm \frac{155 \nu_{0}^{\diamond 3}(\alpha)}{\left(\sigma+C \exp ^{\diamond}\left[4 \varkappa_{1}(\chi, \alpha)\right]\right)^{\diamond \frac{1}{2}}}+\frac{255 \nu_{1}^{\diamond 3}(\alpha)}{\left(\sigma+C \exp ^{\diamond}\left[4 \varkappa_{1}(\chi, \alpha)\right]\right)^{\diamond \frac{3}{4}}} \\
& \left.+\frac{2448 \sigma \nu_{1}^{\diamond 3}(\alpha)}{\left(\sigma+C \exp ^{\diamond}\left[4 \varkappa_{1}(\chi, \alpha)\right]\right)^{\diamond \frac{5}{4}}}\right]
\end{aligned}
$$

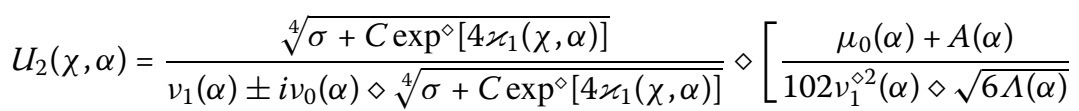

$$
\begin{aligned}
& \pm i \frac{155 v_{0}^{\diamond 3}(\alpha)}{\left(\sigma+C \exp ^{\diamond}\left[4 \varkappa_{1}(\chi, \alpha)\right]\right)^{\diamond \frac{1}{2}}}+i \frac{255 \nu_{1}^{\diamond 3}(\alpha)}{\left(\sigma+C \exp ^{\diamond}\left[4 \varkappa_{1}(\chi, \alpha)\right]\right)^{\diamond \frac{3}{4}}} \\
& \left.+i \frac{2448 \sigma \nu_{1}^{\diamond 3}(\alpha)}{\left(\sigma+C \exp ^{\diamond}\left[4 \varkappa_{1}(\chi, \alpha)\right]\right)^{\diamond \frac{5}{4}}}\right],
\end{aligned}
$$

where

$$
A(\alpha)=\frac{\left(\begin{array}{c}
36 \mu_{0}(\alpha) \diamond v_{0}^{\diamond 2}(\alpha) \diamond v_{1}(\alpha)-180 \mu_{0}^{\diamond 2}(\alpha) \diamond v_{0}(\alpha) \diamond v_{1}(\alpha) \diamond \Delta(\alpha) \\
+12 \mu_{0}^{\diamond 2}(\alpha) \diamond v_{1}(\alpha) \diamond\left(3 v_{0}(\alpha) \diamond \Delta(\alpha)+2 \mu_{0}(\alpha) \diamond \Lambda(\alpha)\right)
\end{array}\right)}{\left(\begin{array}{c}
6\left(\mu_{0}^{\diamond 2}(\alpha) \diamond \Lambda(\alpha)+v_{0}^{\diamond 2}(\alpha)+\mu_{0}(\alpha) \diamond v_{0}(\alpha) \diamond \Delta(\alpha)\right) \\
-\mu_{0}(\alpha) \diamond\left(3 v_{0}(\alpha) \diamond \Delta(\alpha)+2 \mu_{0}(\alpha) \diamond \Lambda(\alpha)\right)
\end{array}\right)}
$$

and

$$
\varkappa_{1}(\chi, \alpha)=\frac{\chi^{\varpi}}{\varpi}+\int_{a}^{\alpha} \frac{\mu_{0}(\eta) \diamond\left(3 v_{0}(\eta) \diamond \Delta(\eta)+2 \mu_{0}(\eta) \diamond \Lambda(\eta)\right)}{6 \eta^{1-\varpi} v_{0}^{\diamond 2}(\eta)} d \eta
$$

such that $\lambda>0$ and $v_{0} \neq 0$.

$$
\begin{aligned}
U_{3}(\chi, \alpha)= & \frac{\sqrt[4]{\sigma+C \exp ^{\diamond}\left[4 \varkappa_{2}(\chi, \alpha)\right]}}{\nu_{1}(\alpha) \pm v_{0}(\alpha) \diamond \sqrt[4]{\sigma+C \exp ^{\diamond}\left[4 \varkappa_{2}(\chi, \alpha)\right]}} \\
& \diamond\left[\frac{\left(v_{0}(\alpha) \diamond \sqrt{9 \Delta^{\diamond 2}(\alpha)-384 \Lambda(\alpha)}-3 v_{0}(\alpha) \diamond \Delta(\alpha)\right)}{8 \Lambda(\alpha)}\right. \\
& \pm \frac{\nu_{1}(\alpha) \diamond \sqrt{3 \Delta^{\diamond 2}(\alpha)-128 \Lambda(\alpha)}-3 v_{1}(\alpha) \diamond \Delta(\alpha)}{8 \Lambda(\alpha) \diamond\left(\sigma+C \exp ^{\diamond}\left[4 \varkappa_{2}(\chi, \alpha)\right]\right)^{\diamond \frac{1}{4}}} \\
& +\frac{\mu_{4}(\alpha) \diamond\left(813 \sigma^{2} v_{0}^{\diamond 2}(\alpha)-8 \mu_{4}^{\diamond 2}(\alpha) \diamond \Lambda(\alpha)\right)}{120 \sigma^{2} \nu_{1}^{\diamond 2}(\alpha) \diamond\left(\sigma+C \exp ^{\diamond}\left[4 \varkappa_{2}(\chi, \alpha)\right]\right)^{\diamond \frac{1}{2}}} \\
& \pm \frac{306 \mu_{4}(\alpha) \diamond v_{0}(\alpha)}{72 \nu_{1}(\alpha) \diamond\left(\sigma+C \exp ^{\diamond}\left[4 \varkappa_{2}(\chi, \alpha)\right]\right)^{\diamond}} \\
& \left.+\frac{\mu_{4}(\alpha)}{\left(\sigma+C \exp ^{\diamond}\left[4 \varkappa_{2}(\chi, \alpha)\right]\right)}\right]
\end{aligned}
$$




$$
\begin{aligned}
U_{4}(\chi, \alpha)= & \frac{\sqrt[4]{\sigma+C \exp ^{\diamond}\left[4 \varkappa_{2}(\chi, \alpha)\right]}}{v_{1}(\alpha) \pm i \nu_{0}(\alpha) \diamond \sqrt[4]{\sigma+C \exp ^{\diamond}\left[4 \varkappa_{2}(\chi, \alpha)\right]}} \\
& \diamond\left[\frac{\left(\nu_{0}(\alpha) \diamond \sqrt{9 \Delta^{\diamond 2}(\alpha)-384 \Lambda(\alpha)}-3 v_{0}(\alpha) \diamond \Delta(\alpha)\right)}{8 \Lambda(\alpha)}\right. \\
& \pm i \frac{\nu_{1}(\alpha) \diamond \sqrt{3 \Delta^{\diamond 2}(\alpha)-128 \Lambda(\alpha)}-3 v_{1}(\alpha) \diamond \Delta(\alpha)}{8 \Lambda(\alpha) \diamond\left(\sigma+C \exp ^{\diamond}\left[4 \varkappa_{2}(\chi, \alpha)\right]\right)^{\diamond \frac{1}{4}}} \\
& +\frac{\mu_{4}(\alpha) \diamond\left(813 \sigma^{2} \nu_{0}^{\diamond 2}(\alpha)-8 \mu_{4}^{\diamond 2}(\alpha) \diamond \Lambda(\alpha)\right)}{120 \sigma^{2} v_{1}^{\diamond 2}(\alpha) \diamond\left(\sigma+C \exp ^{\diamond}\left[4 \varkappa_{2}(\chi, \alpha)\right]\right)^{\diamond \frac{1}{2}}} \\
& \pm i \frac{306 \mu_{4}(\alpha) \diamond v_{0}(\alpha)}{72 v_{1}(\alpha) \diamond\left(\sigma+C \exp ^{\diamond}\left[4 \varkappa_{2}(\chi, \alpha)\right]\right)^{\diamond \frac{3}{4}}} \\
& \left.+\frac{\mu_{4}(\alpha)}{\left(\sigma+C \exp ^{\diamond}\left[4 \varkappa_{2}(\chi, \alpha)\right]\right)}\right]
\end{aligned}
$$

where

$$
\begin{aligned}
& \varkappa_{2}(\chi, \alpha) \\
& \quad=\frac{\chi^{\varpi}}{\varpi}+\int_{a}^{\alpha} \frac{\Delta(\eta, z) \diamond\left(3 \Delta(\eta, z)-\sqrt{9 \Delta^{\diamond 2}(\eta, z)-384 \Lambda(\eta, z)}\right)-64 \Lambda(\eta, z)}{32 \eta^{1-\sigma} \Lambda(\eta, z)} d \eta
\end{aligned}
$$

such that $\lambda \neq 0, \sigma \neq 0, v_{1} \neq 0$, and $\delta^{2} \geq \frac{128 \lambda}{3}$.

$$
\begin{gathered}
U_{5}(\chi, \alpha)=\frac{\left(\begin{array}{c}
528 \mu_{0}(\alpha) \diamond \Lambda(\alpha) \diamond\left(\sigma+C \exp \left[4 \varkappa_{3}(\chi, \alpha)\right]\right)^{\diamond \frac{1}{4}} \\
\pm 66 v_{1}(\alpha) \diamond\left(-3 \Delta(\alpha)+\sqrt{9 \Delta^{\diamond 2}(\alpha)-528 \Lambda(\alpha)}\right)
\end{array}\right)}{\left(\begin{array}{c}
-8 \mu_{0}(\alpha) \diamond \Lambda(\alpha) \diamond\left(3 \Delta(\alpha)+\sqrt{9 \Delta^{\diamond 2}(\alpha)-528 \Lambda(\alpha)}\right) \\
\diamond\left(\sigma+C \exp \left[4 \varkappa_{3}(\chi, \alpha)\right]\right)^{\diamond \frac{1}{4}} \pm 528 v_{1}(\alpha) \diamond \Lambda(\alpha)
\end{array}\right)}, \\
U_{6}(\chi, \alpha, z)=\frac{\left(\begin{array}{c}
528 \mu_{0}(\alpha) \diamond \Lambda(\alpha) \diamond\left(\sigma+C \exp \left[4 \varkappa_{3}(\chi, \alpha)\right]\right)^{\diamond \frac{1}{4}} \\
\pm 66 i \nu_{1}(\alpha) \diamond\left(-3 \Delta(\alpha)+\sqrt{9 \Delta^{\diamond 2}(\alpha)-528 \Lambda(\alpha)}\right)
\end{array}\right)}{\left(\begin{array}{c}
-8 \mu_{0}(\alpha) \diamond \Lambda(\alpha) \diamond\left(3 \Delta(\alpha)+\sqrt{9 \Delta^{\diamond 2}(\alpha)-528 \Lambda(\alpha)}\right) \\
\diamond(\sigma+C \exp [4 \varkappa(\chi, \alpha, z)])^{\diamond \frac{1}{4}} \pm 528 i v_{1}(\alpha) \diamond \Lambda(\alpha)
\end{array}\right)},
\end{gathered}
$$

where

$$
\begin{aligned}
& \varkappa_{3}(\chi, \alpha) \\
& =\frac{\chi^{\varpi}}{\varpi}+\int_{a}^{\alpha} \frac{-3 \Delta^{\diamond 2}(\eta, z)+\Delta(\eta, z) \diamond \sqrt{9 \Delta^{\diamond 2}(\eta, z)-528 \Lambda(\eta, z)}-88 \Lambda(\eta, z)}{32 \eta^{1-\varpi} \Lambda(\eta, z)} d \eta
\end{aligned}
$$

such that $\lambda \neq 0$ and $\delta^{2} \geq \frac{528 \lambda}{9}$.

\section{Discussions and numerical simulations}

This section is devoted to some discussions and numerical simulations to the obtained solutions.

From the equality $\exp ^{\diamond}(\varphi)=\cosh ^{\diamond}(\varphi)+\sinh ^{\diamond}(\varphi)$, our stochastic traveling wave solutions (46), (47), (50), (51), (53), and (54) of Eq. (26) can be handily altered to stochastic solitary 
wave solutions. Especially, the solution $U_{1}(\chi, \alpha)$ can be altered to a solitary stochastic wave solution of the form

$$
\begin{aligned}
U_{1}^{*}(\chi, \alpha)= & \frac{\sqrt[4]{\sigma+C\left(\cosh ^{\diamond}\left[4 \varkappa_{1}(\chi, \alpha)\right]+\sinh ^{\diamond}\left[4 \varkappa_{1}(\chi, \alpha)\right]\right)}}{v_{1}(\alpha) \pm v_{0}(\alpha) \diamond \sqrt[4]{\sigma+C\left(\cosh ^{\diamond}\left[4 \varkappa_{1}(\chi, \alpha)\right]+\sinh ^{\diamond}\left[4 \varkappa_{1}(\chi, \alpha)\right]\right)}} \\
& \diamond\left[\frac{\mu_{0}(\alpha)+A(\alpha)}{102 v_{1}(\alpha)^{\diamond 2} \diamond \sqrt{6 \Lambda(\alpha)}}\right. \\
& \pm \frac{155 v_{0}^{\diamond 3}(\alpha)}{\left(\sigma+C\left(\cosh ^{\diamond}\left[4 \varkappa_{1}(\chi, \alpha)\right]+\sinh ^{\diamond}\left[4 \varkappa_{1}(\chi, \alpha)\right]\right)\right)^{\diamond \frac{1}{2}}} \\
& +\frac{255 v_{1}^{\diamond 3}(\alpha)}{\left(\sigma+C\left(\cosh ^{\diamond}\left[4 \varkappa_{1}(\chi, \alpha)\right]+\sinh ^{\diamond}\left[4 \varkappa_{1}(\chi, \alpha)\right]\right)\right)^{\diamond \frac{3}{4}}} \\
& \left.+\frac{2448 \sigma v_{1}^{\diamond 3}(\alpha)}{\left(\sigma+C\left(\cosh ^{\diamond}\left[4 \varkappa_{1}(\chi, \alpha)\right]+\sinh ^{\diamond}\left[4 \varkappa_{1}(\chi, \alpha)\right]\right)\right)^{\diamond \frac{5}{4}}}\right]
\end{aligned}
$$

where $A(\alpha)$ and $\varkappa_{1}(\chi, \alpha)$ are given in Eqs. (48), (49) and $\Lambda>0$. Also, from the equality $\exp ^{\diamond}(i \varphi)=\cos ^{\diamond}(\varphi)+i \sin ^{\diamond}(\varphi)$, our stochastic traveling wave solutions (46), (47), (50), (51), (53), and (54) of Eq. (26) can be easily altered to stochastic periodic wave solutions. Especially, the solution $U_{1}(\chi, \alpha)$ can be altered to a periodic stochastic wave solution of the form

$$
\begin{aligned}
U_{1}^{* *}(\chi, \alpha)= & \frac{\sqrt[4]{\sigma+C\left(\cos ^{\diamond}\left[4 \varkappa_{1}^{* *}(\chi, \alpha)\right]+i \sin ^{\diamond}\left[4 \varkappa_{1}^{* *}(\chi, \alpha)\right]\right)}}{v_{1}(\alpha) \pm v_{0}(\alpha) \diamond \sqrt[4]{\sigma+C\left(\cos ^{\diamond}\left[4 \varkappa_{1}^{* *}(\chi, \alpha)\right]+i \sin ^{\diamond}\left[4 \varkappa_{1}^{* *}(\chi, \alpha)\right]\right)}} \\
& \diamond\left[\frac{\mu_{0}(\alpha)+A(\alpha)}{102 v_{1}(\alpha)^{\diamond 2} \diamond \sqrt{6 \Lambda(\alpha)}}\right. \\
& \pm \frac{155 v_{0}^{\diamond 3}(\alpha)}{\left(\sigma+C\left(\cos ^{\diamond}\left[4 \varkappa_{1}^{* *}(\chi, \alpha)\right]+i \sin ^{\diamond}\left[4 \varkappa_{1}^{* *}(\chi, \alpha)\right]\right)\right)^{\diamond \frac{1}{2}}} \\
& +\frac{255 v_{1}^{\diamond 3}(\alpha)}{\left(\sigma+C\left(\cos ^{\diamond}\left[4 \varkappa_{1}^{* *}(\chi, \alpha)\right]+i \sin ^{\diamond}\left[4 \varkappa_{1}^{* *}(\chi, \alpha)\right]\right)\right)^{\diamond \frac{3}{4}}} \\
& \left.+\frac{2448 \sigma \nu_{1}^{\diamond 3}(\alpha)}{\left(\sigma+C\left(\cos ^{\diamond}\left[4 \varkappa_{1}^{* *}(\chi, \alpha)\right]+i \sin ^{\diamond}\left[4 \varkappa_{1}^{* *}(\chi, \alpha)\right]\right)\right)^{\diamond \frac{5}{4}}}\right]
\end{aligned}
$$

where $A(\alpha)$ is given in Eq. (48) and

$$
\varkappa_{1}^{* *}(\chi, \alpha)=-i\left(\frac{\chi^{\varpi}}{\varpi}+\int_{a}^{\alpha} \frac{\mu_{0}(\eta) \diamond\left(3 v_{0}(\eta) \diamond \Delta(\eta)+2 \mu_{0}(\eta) \diamond \Lambda(\eta)\right)}{6 \eta^{1-\varpi} \nu_{0}^{\diamond 2}(\eta)} d \eta\right), \quad \Lambda>0
$$

We notice that solutions (46), (47), (50), (51), (53), and (54) of Eq. (26) robustly rely on the given functions $\mu_{0}(\alpha), v_{0}(\alpha), v_{1}(\alpha), \beta_{4}(\alpha), \Delta(\alpha)$, and $\Lambda(\alpha)$. So, for diverse shapes of these functions, we can get diverse solutions of Eq. (26), which can be constructed from Eqs. (46), (47), (50), (51), (53), and (54). We explain this by the following example. We focus our work in this example on the stochastic traveling wave solution $U_{1}$. For the other solutions $U_{2}-U_{6}, U_{1}^{*}$, and $U_{1}^{* *}$, the proceedings are similar. 
Example 5.1 Assume that

$$
\left\{\begin{array}{l}
a=0, \quad v_{0}(\alpha)=a_{0} \mu_{0}(\alpha), \quad v_{1}(\alpha)=a_{1} \mu_{0}(\alpha), \\
\Delta(\alpha)=a_{2} \mu_{0}(\alpha), \quad \Lambda(\alpha)=a_{3} \mu_{0}(\alpha), \quad \mu_{0}(\alpha)=\zeta(\alpha)+a_{4} s^{1-\varpi} W(\alpha),
\end{array}\right.
$$

where $a_{i}(i=0,1, \ldots, a)$ are arbitrary constants, $\zeta(\alpha)$ is a $\varpi$-conformable integrable map on $\mathbb{R}_{+}$, and $W(\alpha)$ is the Gaussian white noise, which is the derivative of the Brownian motion $B(\alpha)$ with respect to $\alpha$. The Hermite transform of $W(\alpha)$ is known as $\widetilde{W}(\alpha, z)=$ $\sum_{i=1}^{\infty} z_{i} \int_{0}^{\alpha} \varrho_{i}(\eta) d \eta[52]$.

Using the identity $\exp ^{\diamond}(B(\alpha))=\exp \left(B(\alpha)-\frac{s^{2}}{2}\right)$ [52], the definition of $\widetilde{W}(\alpha, z)$, and Eq. (46) gives the functional solution of Brownian motion and white noise type of Eq. (26) as follows:

$$
\begin{aligned}
U_{B W}(\chi, \alpha)= & \frac{\sqrt[4]{\sigma+C \exp [4 \Upsilon(\chi, \alpha)]}}{a_{1} \pm a_{0} \sqrt[4]{\sigma+C \exp [4 \Upsilon(\chi, \alpha)]}} \\
& \times\left[\frac{1+A_{1}(\alpha)}{102 a_{1}^{2} \sqrt{6 a_{3}\left(\zeta(\alpha)+a_{4} s^{1-\sigma} W(\alpha)\right)^{\diamond 5}}}\right. \\
& \pm \frac{155 a_{3}^{3}}{(\sigma+C \exp [4 \Upsilon(\chi, \alpha)])^{\frac{1}{2}}}+\frac{255 a_{1}^{3}}{(\sigma+C \exp [4 \Upsilon(\chi, \alpha)])^{\frac{3}{4}}} \\
& \left.+\frac{2448 \sigma a_{1}^{3}}{(\sigma+C \exp [4 \Upsilon(\chi, \alpha)])^{\frac{5}{4}}}\right]
\end{aligned}
$$

where

$$
A_{1}(\alpha)=\frac{\left(\begin{array}{c}
36 a_{2}^{2} a_{1}\left(\zeta(\alpha)+a_{4} s^{1-\varpi} W(\alpha)\right) \\
+\left(24 a_{1} a_{3}-144 a_{0} a_{1} a_{2}\right)\left(\zeta(\alpha)+a_{4} s^{1-\varpi} W(\alpha)\right)^{\diamond 2}
\end{array}\right)}{\left.6 a_{0}^{2}+\left(4 a_{3}+3 a_{0} a_{2}\right)\left(\zeta(\alpha)+a_{4} s^{1-\sigma} W(\alpha)\right)\right)}
$$

and

$$
\Upsilon(\chi, \alpha)=\frac{\chi^{\varpi}}{\varpi}+\frac{3 a_{0} a_{2}+2 a_{3}}{6 a_{0}^{2}}\left[\int_{0}^{\alpha} \frac{\zeta(\eta)}{\eta^{1-\varpi}} d \eta+a_{4}\left(B(\alpha)-\frac{\alpha^{2}}{2}\right)\right] .
$$

For $\varpi=0.01,0.1$, and 1 , the numerical simulation of wave solution (60) is shown in Figs. 1 and 2, when $C=\sigma=a_{1}=a_{3}=1, a_{0}=a_{2}=a_{4}=-1, \zeta(\alpha)=e^{\alpha}, 0 \leq \alpha \leq 4$, and $0 \leq \chi \leq 4$. Figure 1 represents the evolutional behaviors of solution (60) without stochastic effect $(B(\alpha)=W(\alpha)=0)$, and Fig. 2 presents the behavior of solution (60) with the noise effect $B(\alpha)=\operatorname{Random}[0,1] \times \tan (1.7 \alpha)$ and $W(\alpha)=1.7 \operatorname{Random}[0,1] \times \sec ^{2}(1.7 \alpha)$. From Figs. 1 and 2 , it is concluded that the stochastic forcing terms lead to the uncertainty of the traveling wave amplitudes.

\section{Conclusion}

In fact, the stochastic physical models are more sensible than the deterministic models. Thus, right now, we focus the investigation on the SPDEs with conformable derivatives. Foremost, the general Kudryashov method [45] is improved by the novel auxiliary equation (4), which has numerous general solutions depending on the natural number $n$ (see 


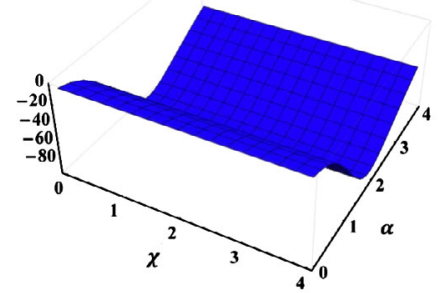

(a)

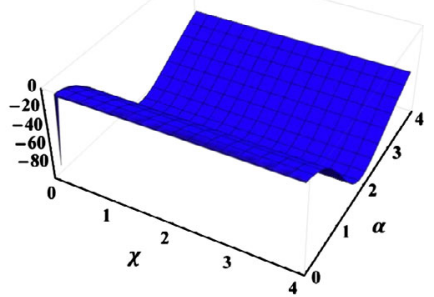

(b)

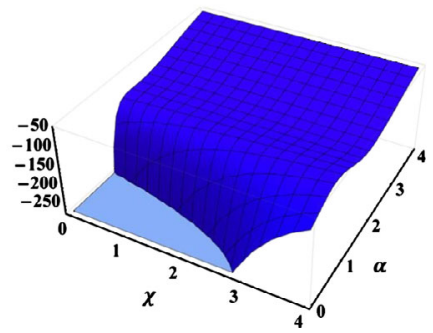

(c)

Figure 1 (a), (b), and (c) 3D plots of solution (60) without the noise effect, when $\varpi=0.01,0.1$, and 1, respectively

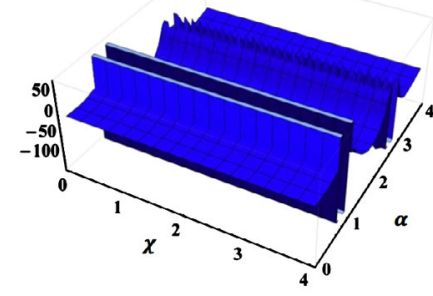

(a)

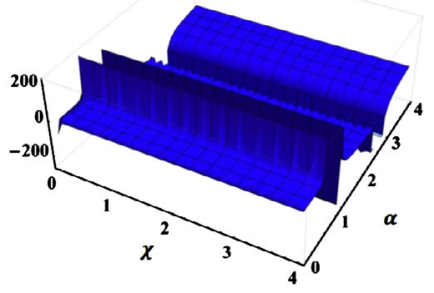

(b)

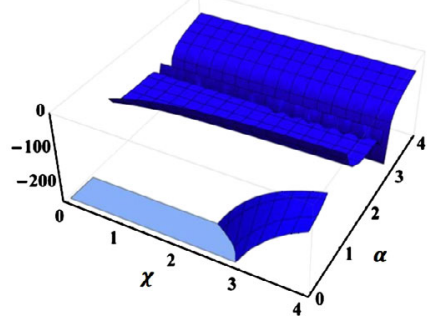

(c)

Figure 2 (a), (b), and (c) 3D plots of solution (60) under the noise effect, when $\varpi=0.01,0.1$, and 1, respectively

Eq. (25)). Thus, a novel technique to build exact solutions for nonlinear evolution equations is obtained. This technique is called the GIKM. The major feature of the GIKM over the others lies in the way that it utilizes an especially clear and powerful algorithm to obtain exact solutions for a large family of nonlinear evolution equations. Also, a large set of exact solutions can be determined effectively on picking the parameters that showed up. Besides, the proposed GIKM generalizes some previous techniques. It depends on im- 
proving the general Kudryashov technique by the general auxiliary equation (4) which has various general solutions. Moreover, we apply the GIKM and white noise topics to construct exact solutions for the Wick-type stochastic combined KdV-mKdV equation with conformable derivatives. Also, numerical simulations with 3D profiles are provided to the obtained exact solutions. Eventually, the overall approach proposed in this paper can be utilized for solving diverse nonlinear evolution equations in physics and engineering, both deterministic and stochastic types.

\section{Appendix}

The system of algebraic equations in $\mu_{i}, v_{j}(i=0,1, \ldots, 5, j=0,1)$, and $\theta$ for the combined $\mathrm{KdV}-\mathrm{mKdV}$ equation

$$
\begin{aligned}
& \mathfrak{X}^{0}: \quad-2 \mu_{1} v_{0}^{2}+\mu_{0}\left(2 v_{0} v_{1}+\mu_{0} v_{0} \delta-2 v_{0}^{2} \omega \theta+\mu_{0}^{2} \lambda\right)=0, \\
& \mathfrak{X}^{1}: \quad-8 \mu_{2} v_{0}^{2}+\mu_{1}\left(2 v_{0} v_{1}+2 \mu_{0} \mu_{1} \delta+2 \mu_{0}^{2} \omega \theta+3 \mu_{0}^{2}\right) \\
& +\mu_{0} v_{1}\left(2 v_{1}+\mu_{0} v_{1} \delta+4 \mu_{0} \omega \theta\right), \\
& \mathfrak{X}^{2}: \quad \mu_{0}\left(3 \mu_{1}^{2} \lambda-2 v_{1}^{2} \omega \theta\right)+\mu_{1}\left(\mu_{1} v_{0} \delta+2 \mu_{0} v_{1} \delta+4 v_{0} v_{1} \omega \theta\right) \\
& +\mu_{2}\left(2 \mu_{0} v_{0} \delta-6 v_{0} v_{1}-2 v_{0}^{2} \omega \theta+3 \mu_{0}^{2} \lambda\right)-18 \mu_{3} v_{0}^{2}=0, \\
& \mathfrak{X}^{3}: \quad-32 \mu_{4} v_{0}^{2}+\mu_{3}\left(2 \mu_{0} v_{0} \delta-22 v_{0} v_{1}-2 v_{0}^{2} \omega \theta+3 \mu_{0}^{2} \lambda\right)+\mu_{2}\left(2 v_{1}^{2}+2 \mu_{1} v_{0} \delta\right. \\
& \left.+2 \mu_{0} v_{1} \delta-4 v_{0} v_{1} \omega \theta+6 \mu_{0} \mu_{1} \lambda\right)+\mu_{1}\left(\mu_{1} v_{1} \delta-2 v_{1}^{2} \omega \theta+\mu_{1}^{2} \lambda\right)=0, \\
& \mathfrak{X}^{4}: \quad-50 \mu_{5} v_{0}^{2}+\mu_{4}\left(46 v_{0} v_{1}+2 \mu_{0} v_{0} \delta-2 v_{0}^{2} \omega \theta+3 \mu_{0}^{2} \lambda\right)+\mu_{3}\left(2 \mu_{1} v_{0} \delta-8 v_{1}^{2}\right. \\
& \left.+2 \mu_{0} v_{1} \delta+4 v_{0} v_{1} \omega \theta+6 \mu_{0} \mu_{1} \lambda\right)+\mu_{2}\left(\mu_{2} v_{0} \delta+2 \mu_{1} v_{1} \delta-2 v_{1}^{2} \omega \theta\right. \\
& \left.+3 \mu_{0} \mu_{2} \lambda+3 \mu_{1}^{2} \lambda\right)+10 v_{0} \sigma\left(\mu_{1} v_{0}-\mu_{0} v_{1}\right)=0, \\
& \mathfrak{X}^{5}: \quad \mu_{5}\left(2 \mu_{0} v_{0} \delta-78 v_{0} v_{1}-2 v_{0}^{2} \omega \theta+3 \mu_{0}^{2} \lambda\right)+\mu_{4}\left(2 \mu_{1} v_{0} \delta-18 v_{1}^{2}+2 \mu_{0} v_{1} \delta\right. \\
& \left.+4 v_{0} v_{1} \omega \theta+6 \mu_{0} \mu_{1} \lambda\right)+\mu_{3}\left(2 \mu_{2} v_{0} \delta+2 \mu_{1} v_{1} \delta+3 \mu_{1}^{2} \lambda-2 v_{1}^{2} \omega \theta+6 \mu_{0} \mu_{2} \lambda\right) \\
& +\mu_{2}\left(\mu_{2} v_{1} \delta+3 \mu_{1} \mu_{2} \lambda+24 v_{0}^{2} \sigma\right)+6 v_{1} \sigma\left(\mu_{1} v_{0}-\mu_{0} v_{1}\right)=0, \\
& \mathfrak{X}^{6}: \quad \mu_{5}\left(-32 v_{1}^{2}+2 \mu_{1} v_{0} \delta-4 v_{0} v_{1} \delta+6 \mu_{0} \mu_{1} \lambda+2 \mu_{0} v_{1} \delta\right)+\mu_{4}\left(2 \mu_{2} v_{0} \delta+2 \mu_{1} v_{1} \delta\right. \\
& \left.-2 v_{1}^{2} \omega \theta+3 \mu_{1}^{2} \lambda+6 \mu_{0} \mu_{2} \lambda\right)+\mu_{3}\left(\mu_{3} v_{0} \delta+2 \mu_{2} v_{1} \delta+6 \mu_{1} \mu_{2} \lambda\right. \\
& \left.+3 \mu_{0} \mu_{3} \lambda+42 v_{0}^{2} \sigma\right)+\mu_{2}\left(30 v_{0} v_{1} \sigma+\mu_{2}^{2} \lambda\right)=0, \\
& \mathfrak{X}^{7}: \quad \mu_{5}\left(2 \mu_{2} \nu_{0} \delta+2 \mu_{1} v_{1} \delta-2 v_{1}^{2} \omega \theta+3 \mu_{1}^{2} \lambda+6 \mu_{0} \mu_{2} \lambda\right)+\mu_{4}\left(2 \mu_{3} \nu_{0} \delta+2 \mu_{2} v_{1} \delta\right. \\
& \left.+6 \mu_{1} \mu_{2} \lambda+6 \mu_{0} \mu_{3} \lambda+64 v_{0}^{2} \sigma\right)+\mu_{3}\left(\mu_{3} v_{1} \delta+3 \mu_{2}^{2} \lambda+3 \mu_{1} \mu_{3} \lambda+62 v_{0} v_{1} \sigma\right) \\
& +10 \mu_{2} v_{1}^{2} \sigma=0, \\
& \mathfrak{X}^{8}: \quad \mu_{5}\left(2 \mu_{3} v_{0} \delta+2 \mu_{2} v_{1} \delta+6 \mu_{1} \mu_{2} \lambda+6 \mu_{0} \mu_{3} \lambda+90 v_{0}^{2} \sigma\right)+\mu_{4}\left(\mu_{4} v_{0} \delta+2 \mu_{3} v_{1} \delta\right. \\
& \left.+3 \mu_{2}^{2} \lambda+3 \mu_{0} \mu_{4} \lambda+6 \mu_{1} \mu_{3} \lambda+102 v_{0} v_{1} \sigma\right)+\mu_{3}\left(3 \mu_{2} \mu_{3} \lambda+24 v_{1}^{2} \sigma\right)=0, \\
& \mathfrak{X}^{9}: \quad \mu_{5}\left(2 \mu_{4} v_{0} \delta+2 \mu_{3} v_{1} \delta+3 \mu_{2}^{2} \lambda+6 \mu_{0} \mu_{4} \lambda+6 \mu_{1} \mu_{3} \lambda+150 v_{0} v_{1} \sigma\right) \\
& +\mu_{4}\left(\mu_{4} v_{1} \delta+6 \mu_{2} \mu_{3} \lambda+3 \mu_{1} \mu_{4} \lambda+42 v_{1}^{2} \sigma\right)+\mu_{3}^{3} \lambda=0, \\
& \mathfrak{X}^{10}: \quad \mu_{5}\left(\mu_{5} v_{0} \delta+2 \mu_{4} v_{1} \delta+6 \mu_{2} \mu_{3} \lambda+6 \mu_{1} \mu_{4} \lambda+3 \mu_{0} \mu_{5} \lambda+64 v_{1}^{2} \sigma\right)
\end{aligned}
$$




$$
\begin{array}{ll} 
& +3 \mu_{4} \lambda\left(\mu_{3}^{3}+\mu_{2} \mu_{4}\right)=0, \\
\mathfrak{X}^{11}: & \mu_{5}\left(\mu_{5} \nu_{1} \delta+3 \mu_{3}^{2} \lambda+6 \mu_{2} \mu_{4} \lambda+3 \mu_{1} \mu_{5} \lambda\right)+3 \mu_{3} \mu_{4}^{2} \lambda=0, \\
\mathfrak{X}^{12}: \quad \mu_{5}\left(6 \mu_{3} \mu_{4} \lambda+3 \mu_{2} \mu_{5} \lambda\right)+\mu_{4}^{3} \lambda=0, \\
\mathfrak{X}^{13}: \quad 3 \mu_{5} \lambda\left(\mu_{4}^{2}+\mu_{3} \mu_{5}\right)=0, \\
\mathfrak{X}^{14}: \quad 3 \mu_{4} \mu_{5}^{2} \lambda=0, \\
\mathfrak{X}^{15}: \quad \mu_{5}^{3} \lambda=0 .
\end{array}
$$

\section{Acknowledgements}

The author extends his appreciation to the Deanship of Scientific Research at King Khalid University for funding his work through General Research Project under grant number (GRP-114-41).

\section{Funding}

This research was funded by King Khalid University under grant number (GRP-114-41).

\section{Abbreviations}

GIKM, General Improved Kudryashov Method; KdV, Korteweg-de Vries; mKdV, Modified Korteweg-de Vries; KdV-mKdV, Korteweg-de Vries and modified Korteweg-de Vries; NODE, Nonlinear Ordinary Differential Equation; NPDE, Nonlinear Partial Differential Equation; PDEs, Partial Differential Equations; SPDEs, Stochastic Partial Differential Equations; 3D, Three-Dimensions.

\section{Availability of data and materials}

The data that support the findings of this study are available from the author upon request.

\section{Competing interests}

The author declares that he has no competing interests.

\section{Authors' contributions}

This research has singular author, who read and approved the final manuscript.

\section{Authors' information}

Full address of the first affiliation is King Khalid University, College of Science, Department of Mathematics, P. O. Box 9004, 61413, Abha, Saudi Arabia.

\section{Publisher's Note}

Springer Nature remains neutral with regard to jurisdictional claims in published maps and institutional affiliations.

Received: 2 December 2019 Accepted: 14 May 2020 Published online: 24 May 2020

\section{References}

1. Wang, M.L.: Solitary wave solutions for variant Boussinesq equations. Phys. Lett. A 199, 169-172 (1995)

2. Hyder, A., Soliman, A.H.: Exact solutions of space-time local fractal nonlinear evolution equations: A generalized conformable derivative approach. Res. Phys. 17, 103135 (2020). https://doi.org/10.1016/j.rinp.2020.103135

3. Wazwaz, A.M.: The tanh-coth method for solitons and kink solutions for nonlinear parabolic equations. Appl. Math. Comput. 188, 1467-1475 (2007)

4. El-Wakil, S.A., El-Labany, S.K., Zahran, M.A., Sabry, R.: Modified extended tanh-function method and its applications to nonlinear equations. Appl. Math. Comput. 161, 403-412 (2005)

5. Liu, X.Q., Jiang, S., Fan, W.B., Liu, W.M.: Soliton solutions in linear magnetic field and time-dependent laser field. Commun. Nonlinear Sci. Numer. Simul. 9, 361-365 (2004)

6. Hirota, R.: Exact solution of Korteweg-de Vries equation for multiple collisions of solitons. Phys. Rev. Lett. 27, 1192-1194 (1971)

7. Tchier, F., Yusuf, A., Aliyu, I.A., Inc, M.: Soliton solutions and conservation laws for lossy nonlinear transmission line equation. Superlattices Microstruct. 107, 320-336 (2017)

8. Inc, M., Yusuf, A., Aliyu, I.A., Baleanu, D.: Soliton structures to some time-fractional nonlinear differential equations with conformable derivative. Opt. Quantum Electron. 50, 20 (2018)

9. Inc, M., Yusuf, A., Aliyu, I.A., Baleanu, D.: Soliton solutions and stability analysis for some conformable nonlinear partial differential equations in mathematical physics. Opt. Quantum Electron. 50, 190 (2018)

10. Bekir, A.: Application of the (G'/G)-expansion method for nonlinear evolution equations. Phys. Lett. A 372, 3400-3406 (2008)

11. Uddin, M.H., Akbar, M.A., Khan, Md.A., Abdul Haque, M.: Close form solutions of the fractional generalized reaction Duffing model and the density dependent fractional diffusion reaction equation. Appl. Comput. Math. 6, 177-184 (2017) 
12. He, J.H., Wu, X.H.: Exp-function method for nonlinear wave equations. Chaos Solitons Fractals 30, 700-708 (2006)

13. Zhao, Y.M.: F-expansion method and its application for finding new exact solutions to the Kudryashov-Sinelshchikov equation. J. Appl. Math. 2013, 895760 (2013)

14. Agarwal, P., Hyder, A., Zakarya, M.: Well-posedness of stochastic modified Kawahara equation. Adv. Differ. Equ. 2020, Article ID 18 (2020)

15. Jarad, F., Uğurlu, E., Abdeljawad, T., Baleanu, D.: On a new class of fractional operators. Adv. Differ. Equ. 2017, Article ID $247(2017)$

16. Benkhettoua, N., Hassania, S., Torres, D.F.M.: A conformable fractional calculus on arbitrary time scales. J. King Saud Univ., Sci. 28, 93-98 (2016)

17. Chung, W.S.: Fractional Newton mechanics with conformable fractional derivative. J. Comput. Appl. Math. 290, 150-158 (2015)

18. Ruzhansky, M., Cho, Y.J., Agarwal, P., Area, I.: Advances in Real and Complex Analysis with Applications. Trends in Mathematics (2018)

19. Agarwal, P., Baleanu, D., Chen, Y., Momani, M.S.: Fractional Calculus: ICFDA 2018, Amman, Jordan, July 16-18. Proceedings in Mathematics and Statistics. Springer, Berlin (2019)

20. Gökdoğan, A., Ünal, E., Çelik, E.: Existence and uniqueness theorems for sequential linear conformable fractional differential equations. Miskolc Math. Notes 17, 267-279 (2016)

21. Qureshi, S., Yusuf, A., Shaikh, A.A., Inc, M.: Transmission dynamics of varicella zoster virus modeled by classical and novel fractional operators using real statistical data. Phys. A, Stat. Mech. Appl. 534, 122-149 (2019)

22. Yépez-Martínez, H., Gómez-Aguilar, J.F.: Optical solitons solution of resonance nonlinear Schrödinger type equation with Atangana's-conformable derivative using sub-equation method. Waves Random Complex Media (2019). https://doi.org/10.1080/17455030.2019.1603413

23. Agarwal, P., Ram, S.: Modelling of transmission dynamics of Nipah virus (Niv): a fractional order approach. Phys. A, Stat. Mech. Appl. 547, 124243 (2020)

24. Agarwal, P., Dragomir, S.S., Jleli, M., Samet, B.: Advances in Mathematical Inequalities and Applications. Trends in Mathematics (2019)

25. Baskonus, H.M., Gómez-Aguilar, J.F.: New singular soliton solutions to the longitudinal wave equation in a magneto-electroelastic circular rod with M-derivative. Mod. Phys. Lett. B 33, 1950251 (2019)

26. Ghanbaria, B., Gómez-Aguilarb, J.F.: New exact optical soliton solutions for nonlinear Schrödinger equation with second-order spatio-temporal dispersion involving M-derivative. Mod. Phys. Lett. B 33, 1950235 (2019)

27. Yusuf, A., Inc, M., Aliyu, A.I.: Fractional solitons for the nonlinear Pochhammer-Chree equation with conformable derivative. J. Coupled Syst. Multiscale Dyn. 6, 158-162 (2018)

28. Agarwal, P.: A Study of New Trends and Analysis of Special Function. LAP Lambert Academic Publishing, Saarbrücken (2013)

29. Agarwal, P., Agarwal, R.P., Ruzhansky, M.: Special Functions and Analysis of Differential Equations. CRC Press, Boca Raton (2020)

30. Wadati, M.: Stochastic Korteweg-de Vries equation. J. Phys. Soc. Jpn. 52, 2642-2648 (1983)

31. Ghany, H.A., Hyder, A., Zakarya, M.: Exact solutions of stochastic fractional Korteweg de-Vries equation with conformable derivatives. Chin. Phys. B 29, 030203 (2020)

32. Soliman, A.H., Hyder, A.: Closed-form solutions of stochastic KdV equation with generalized conformable derivatives. Phys. Scr. 95, 065219 (2020). https://doi.org/10.1088/1402-4896/ab8582

33. Ghany, H.A., Hyder, A., Zakarya, M.: Non-Gaussian white noise functional solutions of $\chi$-Wick-type stochastic KdV equations. Appl. Math. Inf. Sci. 11, 915-924 (2017)

34. Chen, B., Xie, Y.C.: Exact solutions for generalized stochastic Wick-type KdV-mKdV equations. Chaos Solitons Fractals 23, 281-288 (2005)

35. Chen, B., Xie, Y.C.: White noise functional solutions of Wick-type stochastic generalized Hirota-Satsuma coupled KdV equations. J. Comput. Appl. Math. 197, 345-354 (2006)

36. Chen, B., Xie, Y.C.: Periodic-like solutions of variable coefficient and Wick-type stochastic NLS equations. J. Comput. Appl. Math. 203, 249-263 (2007)

37. Hyder, A., Zakarya, M.: Non-Gaussian Wick calculus based on hypercomplex systems. Int. J. Pure Appl. Math. 109, 539-556 (2016)

38. Agarwal, P., Hyder, A., Zakarya, M., AlNemer, G., Cesarano, C., Assante, D.: Exact solutions for a class of Wick-type stochastic (3 + 1)-dimensional modified Benjamin-Bona-Mahony equations. Axioms 8, 134 (2019)

39. Hyder, A., El-Badawy, M.: Distributed control for time-fractional differential system involving Schrödinger operator J. Funct. Spaces 2019, 1389787 (2019)

40. Kudryashov, N.A.: One method for finding exact solutions of nonlinear differential equations. Commun. Nonlinear Sci. Numer. Simul. 17, 2248-2253 (2012)

41. Ege, S.M., Misirli, E.: The modified Kudryashov method for solving some fractional-order nonlinear equations. Adv. Differ. Equ. 2014, Article ID 135 (2014). https://doi.org/10.1186/1687-1847-2014-135

42. Zayed, E.M.E., Alurrfi, K.A.E.: The modified Kudryashov method for solving some seventh order nonlinear PDEs in mathematical physics. World J. Model. Simul. 11, 308-319 (2015)

43. Kilicman, A., Silambarasan, R.: Modified Kudryashov method to solve generalized Kuramoto-Sivashinsky equation. Symmetry 10, $527(2018)$

44. Kumar, D., Seadawy, A.R., Joardar, A.K.: Modified Kudryashov method via new exact solutions for some conformable fractional differential equations arising in mathematical biology. Chin. J. Phys. 56, 75-85 (2018)

45. Islam, M.S., Khan, K.A., Arnous, H.: Generalized Kudryashov method for solving some (3+1)-dimensional nonlinear evolution equations. New Trends Math. Sci. 3, 46-57 (2015)

46. Mahmud, F., Samsuzzoha, M., Akbar, M.A.: The generalized Kudryashov method to obtain exact traveling wave solutions of the PHI-four equation and the Fisher equation. Results Phys. 7, 4296-4302 (2017)

47. Islam, N., Khan, K., Islam, M.H.: Travelling wave solution of Dodd-Bullough-Mikhailov equation: a comparative study between generalized Kudryashov and improved F-expansion methods. J. Phys. Commun. 3, 055004 (2019)

48. Rahman, M.M., Habib, M.A., Ali, H.M.S., Miah, M.M.: The generalized Kudryashov method: a renewed mechanism for performing exact solitary wave solutions of some NLEEs. J. Mech. Contin. Math. Sci. 14, 323-339 (2019) 
49. Abdus Salam, M., Habiba, U.: Application of the improved Kudryashov method to solve the fractional nonlinear partial differential equations. J. Appl. Math. Phys. 7, 912-920 (2019)

50. Khalil, R., Al Horani, M., Yousef, A., Sababheh, M.A.: A new definition of fractional derivative. J. Comput. Appl. Math. 246, 65-70 (2014)

51. Çenesiz, Y., Baleanu, D., Kurt, A., Tasbozan, O.: New exact solutions of Burgers type equations with conformable derivative. Waves Random Complex Media 27, 103 (2017)

52. Holden, H., Øksendal, B., Ubøe, J., Zhang, T.: Stochastic Partial Differential Equations. Springer, Berlin (2010)

53. Ghany, H.A., Hyder, A.: Soliton solutions for Wick-type stochastic fractional KdV equations. Int. J. Math. Anal. 7, 2199-2208 (2013)

Submit your manuscript to a SpringerOpen ${ }^{\odot}$ journal and benefit from:

- Convenient online submission

- Rigorous peer review

- Open access: articles freely available online

- High visibility within the field

- Retaining the copyright to your article

Submit your next manuscript at $\boldsymbol{\triangleright}$ springeropen.com 